\title{
How can nanoparticles be used in sentinel node detection?
}

\author{
"Sentinel node biopsy is the standard of care for axillary \\ staging in breast cancer patients with a clinically and \\ radiologically normal axilla."
}

First draft submitted: 12 April 2017; Accepted for publication: 21 April 2017; Published online: 16 June 2017

Keywords: antibody labeling • axillary staging • axillary surgery $\bullet$ magnetic nanoparticles

- multimodal probes $\bullet$ sentinel node biopsy (SNB)

Sentinel node biopsy (SNB) is the standard of care for axillary staging in breast cancer patients with a clinically and radiologically normal axilla. The concept is based upon the exclusion of metastases in the first draining lymph node(s) from the primary tumor. The current practice utilizes a radiolabeled tracer and blue dye (combined technique) with reported sentinel node identification rates of $96.4 \%$, and a false negative rate of $7.3 \%$ in a large meta-analysis [1]. Although successful, this technique is limited by its dependence upon radioisotopes - with their inherent strict requirements for handling, transport, disposal and close access to nuclear medicine facilities. Blue dye is also associated with prolonged skin staining and reports of anaphylaxis in $0.9 \%$ of patients [2]. These limitations have resulted in the on-going development of alternative techniques for SNB [3,4].

\section{Magnetic technique for SNB}

One of the most promising radioisotopeindependent techniques for SNB comprises an interstitial injection of superparamagnetic iron oxide (SPIO) nanoparticles, which are identified intraoperatively with a handheld magnetometer and through visual brownblack staining of nodes. The magnetic technique has now been evaluated in a number of studies and confirmed on meta-analysis to be noninferior to the radioisotope-dependent technique in breast cancer - with sentinel node identification rates of 97.1 and $96.8 \%$, respectively [3] - and demonstrated technical benefits over other novel techniques [4]. The noninferiority of the magnetic technique has also been demonstrated in the staging of malignant melanoma [5] and has been applied to a range of solid cancers in small cohort series, which has demonstrated its feasibility in the staging of these malignancies $[6-8]$. What is still lacking is randomized controlled trial evidence, before the magnetic technique can be considered as an alternative to the standard technique.

\section{Selective axillary surgery}

The current trend within axillary management in breast cancer is toward quantification of the axillary metastatic burden. The identification of low axillary burden has been shown to allow avoidance of formal axillary node clearance, without compromising overall and disease-free survival at long-term follow-up [9]. This evidence is leading to the concept of 'selective axillary surgery', which would allow targeted excision of only metastatically involved nodes. This relies upon improved preoperative imaging combined with novel techniques to guide surgeons intraoperatively, to involved nodes.

\section{Multimodal MRI for assessing nodal involvement}

Magnetic nanoparticles have been used for axillary staging noninvasively with MRI and demonstrated a sensitivity and specificity for

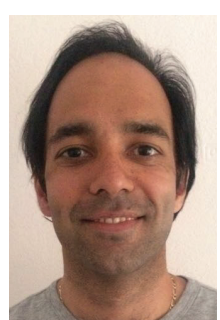

Muneer Ahmed

Division of Cancer Studies, King's College London, Guy's Hospital Campus, Great Maze Pond, London SE1 9RT, UK

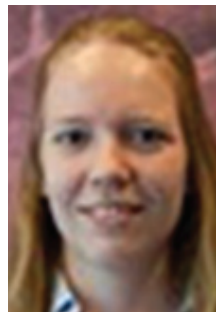

Mirjam CL Peek

Division of Cancer Studies, King's College London, Guy's Hospital Campus, Great Maze Pond, London SE1 9RT, UK

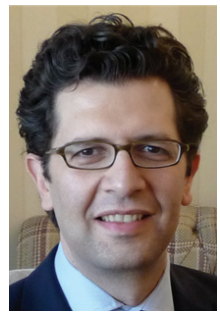

Michael Douek

Author for correspondence: Division of Cancer Studies, King's College London, Guy's Hospital Campus, Great Maze Pond, London SE1 9RT, UK michael.douek@kcl.ac.uk

Future $\because$ Medicine part of 
the detection of axillary involvement of 98 and 96\%, respectively [10]. A limitation to implementation of this approach is the elevated false positive rate compared with SNB [11]. This has led to the development of dual probes to allow for the benefits of multimodal imaging. Magnetic tracers have been conjugated with radiolabeled bisphosphonates in order to allow simultaneous, excellent anatomical visualization on MRI and computerized tomography (CT) scanning, in addition to functional categorization on positron emission tomography [12]. Triple-modal imaging magnetic nanocapsules, encapsulating hydrophobic SPIO to magnetically target solid tumors after intravenous administration have been developed [13]. Within a murine model, the magnetic polymeric nanocapsules demonstrated a twofold increase in tumor uptake when a static magnetic field was applied [13]. The probes allowed fluorescence, MRI and nuclear imaging to be performed, providing complementary information on the spatial distribution of the nanocarrier within the tumor. Consequently, multimodal probes, which target metastatic foci have been developed. These have included fluorescent-ferrite beads containing both magnetic iron oxide and fluorescent europium complexes, which have been labeled with antibodies to a range of clinically relevant antigens (BNP, PSA, EGFR, CA19-9 and HB-8509). The activation of these particles by application of a magnetic field allows for immunohistochemical staining and enhanced intensity of fluorescence and focal quantification of antigen, resulting in targeted therapies and diagnostics [14].

\section{"The use of multimodal probes allows for optimization of preoperative imaging to characterize lymph nodes and quantify nodal burden."}

The use of multimodal probes allows for optimization of preoperative imaging to characterize lymph nodes and quantify nodal burden. The multimodal labeling also allows the surgeon to utilize different techniques to localize involved nodes and target them precisely. Open surgery could be directed with a handheld magnetometer to identify 'hot spots' of magnetic tracer accumulation and hence metastatically involved nodes. Minimally invasive, percutaneous techniques could be used to target the magnetic tracer accumulation (nodes) under MRI-guided vision and excise specimens en bloc. Alternatively, completely noninvasive techniques using MRI-guided high-intensity focused ultrasound could be applied to the same targets of focal iron accumulation and ablate the specimens without excision. This could equally be potentially achieved by the application of an alternating magnetic field to induce targeted magnetic hyperthermia.
It has been demonstrated from preclinical porcine models [15] that only $2 \%$ of injected SPIO reaches the draining sentinel nodes when the clinical volume of 2 $\mathrm{ml}$ (54 mg iron oxide) of magnetic tracer is injected interstitially. Consequently, evidence has emerged from the largest trial of the magnetic technique that this retention of iron can produce void artifacts on MRI and that in some patients this can be prolonged [16]. This area requires prospective evaluation to confirm these findings but it is essential that on-going research attempts to reduce iron retention at injection sites. The injection of lower volumes $(0.5 \mathrm{ml})$ intratumorally was explored clinically but resulted in low sentinel node identification rates compared with the standard combined technique (85 vs 97\%) [17]. Porcine models demonstrated a significant inverse relationship between increasing injection volumes and percentage iron uptake (relative to injected dose) by sentinel nodes $(p<0.001)$ [15]. Future work is needed to evaluate lower volume interstitial injections and the feasibility of intravenous administration.

\section{Future perspective}

The impact of increasingly conservative axillary management in breast cancer has increased the importance of preoperative staging using ultrasound. Trials are assessing whether a negative axilla on ultrasound may eliminate the need for SNB completely. It is very likely that in future, pending the results of ongoing trials, that the clinical indication for SNB may be reduced. However, accurate staging will still be required in those patients who possess an abnormal preoperative axillary ultrasound. This will be particularly the case in patients who are receiving primary systemic therapy and who will be assessed for their response before undergoing definitive surgery. It has been shown that when this cohort of patients undergoes SNB, they have an elevated false negative rate $(12 \%)$, which is potentially lowered on removal of three or more nodes (9\%) [18]. If the suspicious nodes are clipped at ultrasound and removed at SNB, then the false negative rate may fall further to less than $2 \%$ [19]. The application of a solid ferromagnetic marker - introduced $[19,20]$ into the suspicious nodes - which could subsequently be excised under intraoperative guidance of a handheld magnetometer, would be clinically relevant and avoid technical and logistical difficulties associated with radioactive markers or wires. Both removal of more nodes and clipping of nodes have not been confirmed in subsequent clinical trials as yet.

The use of SNB is in an advanced stage within breast cancer compared with other solid cancers. This has resulted in the emergence of the necessity to quan- 
tify axillary lymph node burden in determining management rather than simply confirming the presence or absence of metastatic involvement. This places nanomedicine in a unique position to allow the emergence of targeted imaging and therapies. These therapies will allow the development of minimally invasive, selective axillary surgery, targeting metastatic nodes alone and provide more personalized treatments for cancer patients of the future.

\section{References}

1 Kim T, Giuliano AE, Lyman GH. Lymphatic mapping and sentinel lymph node biopsy in early-stage breast carcinoma: a metaanalysis. Cancer 106(1), 4-16 (2006).

2 Barthelmes L, Goyal A, Newcombe RG, McNeill F, Mansel RE. Adverse reactions to patent blue V dye - the NEW START and ALMANAC experience. Eur. J. Surg. Oncol. 36(4), 399-403 (2010).

3 Zada A, Peek MC, Ahmed M et al. Meta-analysis of sentinel lymph node biopsy in breast cancer using the magnetic technique. Br. J. Surg 103(11), 1409-1419 (2016).

4 Ahmed M, Purushotham AD, Douek M. Novel techniques for sentinel lymph node biopsy in breast cancer: a systematic review. Lancet Oncol. 15(8), e351-e362 (2014).

5 Anninga B, White $\mathrm{SH}$, Moncrieff $\mathrm{M}$ et al. Magnetic technique for sentinel lymph node biopsy in melanoma: the MELAMAG Trial. Ann. Surg. Oncol. 23(6), 2070-2078 (2016).

6 Ahmed M, Douek M. The role of magnetic nanoparticles in the localization and treatment of breast cancer. BioMed. Res. Int. 2013, 281230 (2013).

7 Nakagawa T, Minamiya Y, Katayose Y et al. A novel method for sentinel lymph node mapping using magnetite in patients with non-small cell lung cancer. J. Thorac. Cardiovasc. 126(2), 563-567 (2003).

8 Winter A, Woenkhaus J, Wawroschek F. A novel method for intraoperative sentinel lymph node detection in prostate cancer patients using superparamagnetic iron oxide nanoparticles and a handheld magnetometer: the initial clinical experience. Ann. Surg. Oncol. 21(13), 4390-4396 (2014).

9 Giuliano AE, Ballman K, McCall L et al. Locoregional recurrence after sentinel lymph node dissection with or without axillary dissection in patients with sentinel lymph node metastases: long-term follow-up from the American College of Surgeons Oncology Group (Alliance) ACOSOG Z0011 Randomized Trial. Ann. Surg. 264(3), 413-420 (2016).

10 Harnan SE, Cooper KL, Meng Y et al. Magnetic resonance for assessment of axillary lymph node status in early breast cancer: a systematic review and meta-analysis. Eur. J. Surg. Oncol. 37(11), 928-936 (2011).

\section{Financial \& competing interests disclosure}

The authors have no relevant affiliations or financial involvement with any organization or entity with a financial interest in or financial conflict with the subject matter or materials discussed in the manuscript. This includes employment, consultancies, honoraria, stock ownership or options, expert testimony, grants or patents received or pending, or royalties.

No writing assistance was utilized in the production of this manuscript.

11 Meng Y, Ward S, Cooper K, Harnan S, Wyld L. Costeffectiveness of MRI and PET imaging for the evaluation of axillary lymph node metastases in early stage breast cancer. Eur. J. Surg. Oncol. 37(1), 40-46 (2011).

12 Torres Martin de Rosales R, Tavare R, Glaria A, Varma G, Protti A, Blower PJ. $\left({ }^{99} \mathrm{~m}\right)$ Tc-bisphosphonate-iron oxide nanoparticle conjugates for dual-modality biomedical imaging. Bioconjug Chem. 22(3), 455-465 (2011).

13 Bai J, Wang JT, Rubio $\mathrm{N}$ et al. Triple-modal imaging of magnetically-targeted nanocapsules in solid tumours in vivo. Theranostics 6(3), 342-356 (2016).

14 Sakamoto S, Omagari K, Kita Y et al. Magnetically promoted rapid immunoreactions using functionalized fluorescent magnetic beads: a proof of principle. Clin. Chem. 60(4), 610-620 (2014).

15 Ahmed M, Anninga B, Pouw JJ et al. Optimising magnetic sentinel lymph node biopsy in an in vivo porcine model. Nanomedicine 11(4), 993-1002 (2015).

16 Huizing E, Anninga B, Young P, Monnypenny I, HallCraggs M, Douek M. Analysis of void artefacts in postoperative breast MRI due to residual SPIO after magnetic SLNB in SentiMAG Trial participants. Eur. J. Surg. Oncol. 41(6), S18 (2015).

17 Ahmed M, Anninga B, Goyal S, Young P, Pankhurst QA, Douek M. Magnetic sentinel node and occult lesion localization in breast cancer (MagSNOLL Trial). Br. J. Surg. 102(6), 646-652 (2015).

18 Boughey JC, Suman VJ, Mittendorf EA et al. Sentinel lymph node surgery after neoadjuvant chemotherapy in patients with node-positive breast cancer: the ACOSOG Z1071 (Alliance) clinical trial. JAMA 310(14), 1455-1461 (2013).

19 Caudle AS, Yang WT, Krishnamurthy $S$ et al. Improved axillary evaluation following neoadjuvant therapy for patients with node-positive breast cancer using selective evaluation of clipped nodes: implementation of targeted axillary dissection. J. Clin. Oncol. 34(10), 1072-1078 (2016).

20 Donker M, Straver ME, Wesseling J et al. Marking axillary lymph nodes with radioactive iodine seeds for axillary staging after neoadjuvant systemic treatment in breast cancer patients: the MARI procedure. Ann. Surg. 261(2), 378-382 (2015). 